\title{
Critical Aspects in Calibration of ESD Generators
}

\author{
A. Morando ${ }^{1}$, M. Borsero ${ }^{2}$, A. Sardi $^{2}$, G. Vizio $^{2}$ \\ ${ }^{1}$ Politecnico di Torino, Dipartimento di Ingegneria Elettrica, C.so Duca degli Abruzzi 24, 10129 Torino (Italy), \\ +390113919830, andrea.morando@polito.it \\ 2 Istituto Nazionale di Ricerca Metrologica, INRIM, Strada delle Cacce 91, 10135 Torino (Italy), +390113919348, \\ m.borsero@inrim.it
}

\begin{abstract}
Electrostatic discharge (ESD) immunity tests are widely carried out in order to assess the immunity level of electrical and electronic equipment. ESD is a severe source of interference which can produce damage, upset or failures in any electronic system. A careful calibration procedure is required to assure that the ESD generator parameters fulfill the standard specifications. In this paper, the main critical aspects involved in the calibration measurement setup are dealt with and a simple modeling support is given. On the basis of the obtained results, some useful conclusions and practical suggestions are finally drawn.
\end{abstract}

Keywords: Electrostatic discharges, calibration, electromagnetic compatibility, electromagnetic measurements, modeling, immunity.

\section{INTRODUCTION}

$\mathrm{T}$ HE EUROPEAN Directive 2004/108/EC [1] on electromagnetic compatibility (EMC) dictates that a lot of tests on electrical and electronic equipment should be performed in order to guarantee both low electromagnetic emission levels and a sufficient degree of immunity against different kinds of disturbance in the environment. One of the most severe immunity tests concerns the Electro-Static Discharges (ESDs), which can momentarily disturb the operation of a system or provoke failures and even cause permanent damage. The ESD on electronic devices can occur when a device becomes charged by triboelectrification and approaches another conductive element or when a human charged by triboelectrification handles the device. Charge accumulation by triboelectrification or induction process is at the origin of any ESD event. The effects produced by ESD are usually observed by separating conducted interference (i.e. direct effect) due to the direct injection of the discharge current in the victim device, and radiated interference (i.e. indirect effect) related to the coupling with the electromagnetic field radiated by the ESD event.

During the last years, a great effort has been made by the scientific community to establish ESD standard procedures. To this aim, an important role has been played by several studies on ESD current waveform, focusing the attention on the amplitude and rise-time variations due to different voltages, approach speeds, relative humidity and electrode shapes [2], [3].

The immunity test has to comply with the IEC and ISO standards [4], [5], where immunity requirements and test methods are fixed for equipment which must withstand ESD, either directly or indirectly. The standards consider two test methods: the contact discharge (CD) and the air discharge (AD) methods. In the $\mathrm{CD}$ method, the generator discharge tip is kept in direct contact with the equipment under test (EUT) and the discharge is fired by closing a high-voltage switch. In the AD method, the discharge tip approaches the EUT keeping the switch closed, until a discharge in the air gap is obtained.
Starting with the actual discharge characteristics, ESD generators are designed to provide current waveforms similar to those generated by the human body.

To improve the reproducibility of the results, the traceability of ESD generators, obtained by periodical calibrations, has to be accomplished. The many years of experience of INRIM (Istituto Nazionale di Ricerca Metrologica) in calibration activity and an international comparison [6] demonstrate that considerable differences may occur in the results, especially when generators of different manufacturers are used. This fact has important effects with regard to the decisions to be taken as to the compliance with the EMC Directive requirements.

In the present work, theoretical and experimental investigations have been made about the calibration procedure of ESD generators. In addition to calibration, testing laboratories must implement simplified "verification" techniques to check the actual performance of their measuring equipment and the fulfillment of the requirements. However, these verification methods are not the subject of this paper.

\section{MEASUREMENT SET-UP}

The immunity standards [4], [5] identify the CD method as the preferred test. As far as the CD method is concerned, the EMC standards recommend to check the following parameters related to the discharge current waveform (Table $1)$ : the first peak value $\left(I_{p}\right)$, the rise time $\left(t_{r}\right)$ and the current values at $30 \mathrm{~ns}\left(I_{30}\right)$ and $60 \mathrm{~ns}\left(I_{60}\right)$.

As far as the AD method is concerned, it shall be used where contact discharges cannot be applied. The same standards do not recommend any specific calibration method: only the tolerance of the charge voltage is specified and set equal to $5 \%$ of the nominal value.

The measurement setup employed in the calibration of ESD generators, according to the CD method, is made of several blocks (Fig.1): the ESD generator can be modeled by means of a basic equivalent circuit [4], consisting of a high voltage DC supply, a charging resistor $R_{c}$, an energystorage capacitor $C_{s}$, a discharging resistor $R_{d}$, a discharge switch $S$ and a discharge return connection. 
The generator is often equipped with a discharge network plug-in, which allows different choices for $C_{s}$ and $R_{d}$, whose values range from $100 \mathrm{pF}$ to $330 \mathrm{pF}$ and from $330 \Omega$ to 2000 $\Omega$, respectively. The test level is defined as the charge voltage of the capacitor $C_{s}$. The levels fixed by the EMC standards range from $2 \mathrm{kV}$ to $25 \mathrm{kV}$.

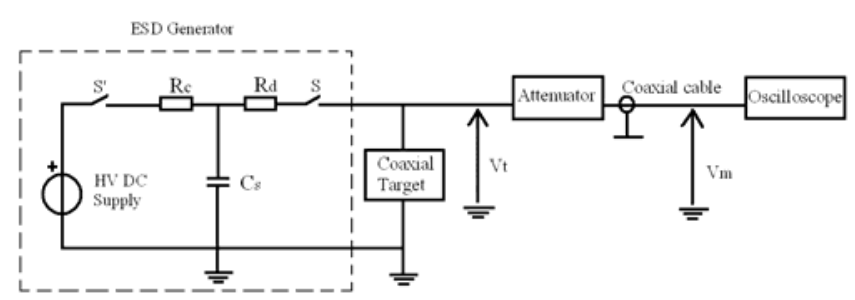

Fig.1. Measurement system for the ESD generator calibration-CD method.

The discharge current return connection between the generator and the reference ground is made of a ground strap cable. In the calibration set-up the current has to be detected by means of a small-inductance coaxial shunt (the "target" in Fig.1), together with an oscilloscope with a minimum bandwidth of $2 \mathrm{GHz}$. Annex $\mathrm{B}$ of the IEC standard [4] specifies the requirements to build a target, together with the details of the transfer impedance of the target-attenuator-cable chain. The target has to be made with a low resistance of $2 \Omega$ on the test side. The target is mounted in the center of a vertical metal plane. An attenuator and a radiofrequency coaxial cable are used to improve the impedance matching and to carry the signal to the measuring instrument. The digital oscilloscope is employed to analyze the discharge current waveform in the time domain. It is placed inside a Faraday cage to prevent it from the effects of the electromagnetic field radiated by the ESD.

The ESD events are quite non-repeatable. The calibration of the ESD generator should be carried out carefully in order to verify if the ESD simulator fulfills the requirements in Table 1. Moreover, [4] fixes the limit values for the uncertainty associated to the calibration of the different waveform parameters. A deep and detailed analysis of the whole measurement chain has been dealt with by [7], where the influence of the target parameters and dynamic properties of the oscilloscope on the "current metrics" are highlighted. In order to provide some uncertainty values, reference [2] specifies the following typical uncertainty contributions $(2 \sigma)$ derived from many reports by accredited calibration laboratories: $8 \%$ for the current peak value and $5-7 \%$ for the rise time in the CD mode.

\section{DEVELOPMENTS AND IMPROVEMENTS}

INRIM has developed a system to calibrate ESD generators. A coaxial shunt, used in the CD calibration test, has been characterized accurately and its frequency response has been evaluated by means of a vector network analyzer up to 4 $\mathrm{GHz}$ (Table 2 and 3). Table 2 shows the good agreement between the measured DC resistance and the requirement of the Annex $\mathrm{C}$ of [4]. The insertion loss values, reported in Table 3 , are well within $\pm 0.5 \mathrm{~dB}$ over the whole frequency range from $10 \mathrm{kHz}$ to $4 \mathrm{GHz}$, fulfilling again the IEC standard specifications.

Tab.1. CD current waveform parameters and tolerances (IEC 61000-4-2).

\begin{tabular}{|c|c|c|c|c|c|}
\hline Level & $\begin{array}{c}\text { Indicated } \\
\text { voltage } \\
\mathrm{kV}\end{array}$ & $\begin{array}{c}\text { First peak } \\
\text { current of } \\
\text { discharge } \\
( \pm 15 \%) \\
\text { A } \\
\end{array}$ & $\begin{array}{c}\text { Rise } \\
\text { time } t_{r} \\
( \pm 25 \%) \\
\text { ns }\end{array}$ & $\begin{array}{c}\text { Current } \\
( \pm 30 \%) \\
\text { at } 30 \text { ns } \\
\text { A }\end{array}$ & $\begin{array}{c}\text { Current } \\
( \pm 30 \%) \\
\text { at } 60 \mathrm{~ns} \\
\mathrm{~A}\end{array}$ \\
\hline 1 & 2.0 & 7.5 & 0.8 & 4.0 & 2.0 \\
\hline 2 & 4.0 & 15 & 0.8 & 8.0 & 4.0 \\
\hline 3 & 6.0 & 22.5 & 0.8 & 12.0 & 6.0 \\
\hline 4 & 8.0 & 30 & 0.8 & 16.0 & 8.0 \\
\hline
\end{tabular}

Tab. 2. Target input DC resistance.

\begin{tabular}{cc}
\hline Nominal value: & $2.000 \Omega$ \\
Measured value: & $1.971 \Omega$ \\
Uncertainty: & $0.006 \Omega$ \\
\hline
\end{tabular}

Tab. 3. Insertion Loss as a function of frequency.

\begin{tabular}{ccc}
\hline Frequency $(\mathrm{MHz})$ & Insertion Loss $(\mathrm{dB})$ & Uncertainty $(\mathrm{dB})$ \\
\hline 0.01 & 42.67 & 0.40 \\
\hline 1 & 42.78 & 0.29 \\
\hline 100 & 42.93 & 0.28 \\
\hline 1000 & 43.08 & 0.28 \\
\hline 2000 & 43.19 & 0.28 \\
\hline 4000 & 42.35 & 0.33 \\
\hline
\end{tabular}

With regard to the $\mathrm{AD}$ method, the generator calibration can be performed by measuring the charge voltage through high input impedance systems. This operation is carried out by closing the switch S' (see Fig. 1) and keeping the generator tip in contact with the input terminals of a high impedance probe, whose output voltage is read by a multimeter and its waveform is drawn on a digital oscilloscope (Fig.2). After an initial transient phase, the output voltage reaches a steady-state DC value, as shown in Fig.3. Since the input impedance is very high but finite, the measurement chain exhibits a load effect. The aim is to find out the minimum input impedance that guarantees an acceptable load effect, compared to the required tolerances.

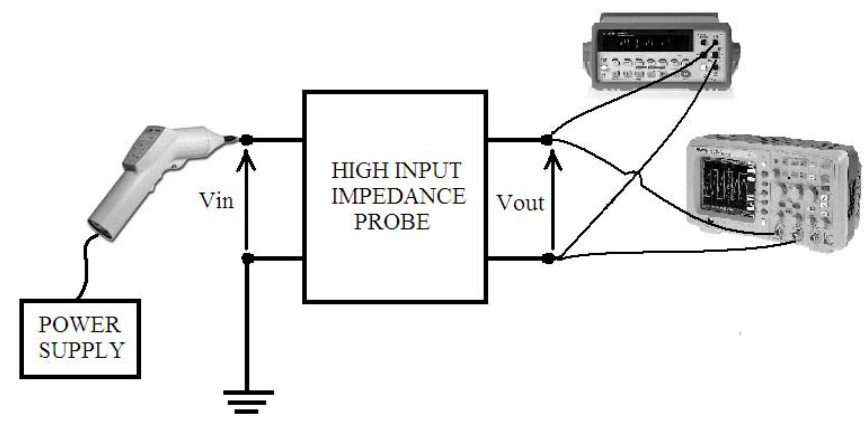

Fig.2. Measurement system for the ESD generator calibration-AD method. 
Many high voltage probes for DC measurements with an input impedance of $1 \mathrm{G} \Omega$ and a nominal divider ratio $r$ $\left(r=\mathrm{V}_{\text {in }} / \mathrm{V}_{\text {out }}\right.$ ) equal to $10^{3}$ have been tested. By using a $40 \mathrm{kV}$ probe coupled with a digital sampling oscilloscope (DSO), a strong load effect of about $15 \%$ (at a nominal voltage of 4 $\mathrm{kV}$ ) can be detected (Fig.3). When the input terminals of the probe are connected to the generator output, an initial stepup of the voltage is followed by a slow decay.

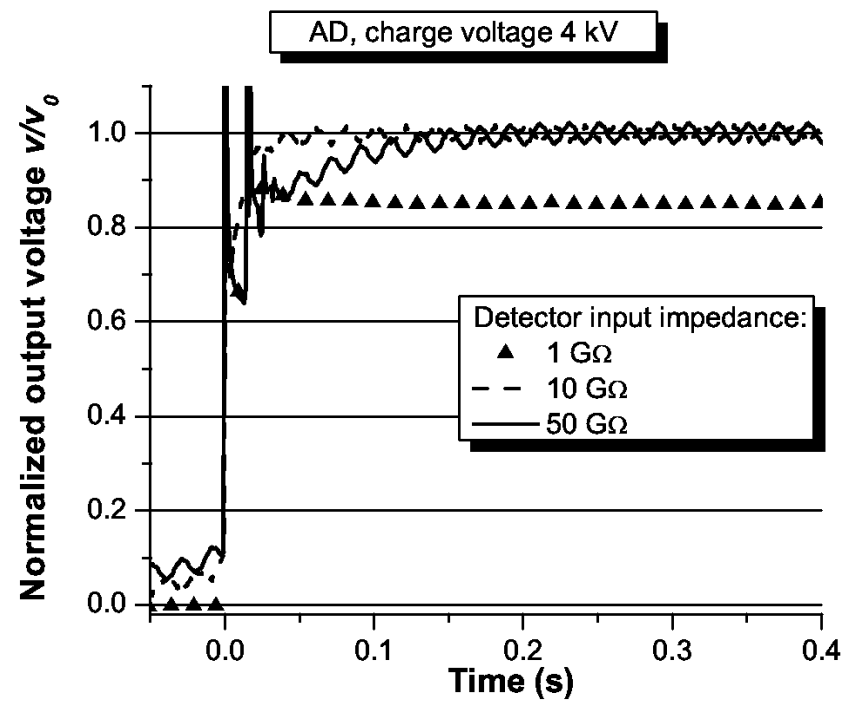

Fig.3. Comparison of the load effect on a $4 \mathrm{kV}$ charge voltage, by using a $1 \mathrm{G} \Omega$, a $10 \mathrm{G} \Omega$ and a $50 \mathrm{G} \Omega$ divider.

The values shown in Fig. 3 are normalized with respect to the rated voltage value $v_{0}(4 \mathrm{kV})$. This effect has been proved through measurements for several voltage levels (from 1 to $15 \mathrm{kV}$ ) and an analogous behavior has been found at each step. In order to reduce this effect, a resistive voltage divider with an input impedance of $10 \mathrm{G} \Omega$ and $r$ equal to $10^{4}$ has been built as a voltage converter device [8]. The voltage waveform detected by using the INRIM $10 \mathrm{G} \Omega$ divider, coupled with a DSO, shows a reduced load effect of about $0.8 \%$ (Fig.3).

To further reduce the voltage drop, a new resistive voltage divider with a $50 \mathrm{G} \Omega$ input impedance has been designed. Ten 5 G $\Omega$ high voltage (HV) resistors have been selected to obtain a uniform voltage distribution along the divider chain (Fig.4). The low voltage resistance consists of two resistors connected in parallel. The divider ratio $\left(r=10^{4}\right)$ has been chosen according to the high input impedance/high accuracy range of the multimeter. Both high and low voltage resistors have been placed in a PMMA cylinder. Two electrodes mounted on the top and bottom of the cylinder avoid the occurrence of the corona discharge. As the HV resistor values can be affected by surface conductivity, the humidity in the cylinder is lowered using silica gel. The linearity is ensured by a comparison with the INRIM HV DC reference measuring system. As expected on the basis of the $10 \mathrm{G} \Omega$ divider results, a load effect of about $0.1 \%$ is obtained with the $50 \mathrm{G} \Omega$ device (Fig.3).

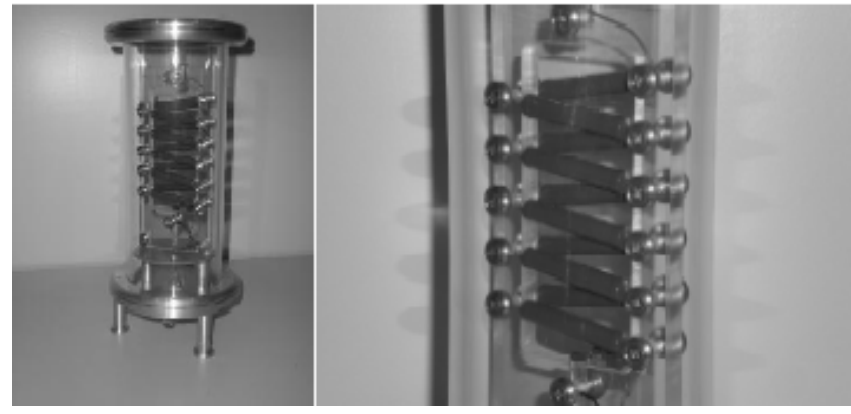

Fig.4. Details of the INRIM $50 \mathrm{G} \Omega$ voltage divider.

It can be inferred that a satisfactory reduction of the load effect is obtained by means of a voltage divider with at least $10 \mathrm{G} \Omega$ input impedance.

\section{CRITICAL ASPECTS - CD METHOD}

This analysis has been performed whilst paying special attention to the below items with regard to the current waveform and its parameters. Two models of ESD generators by EM TEST manufacturer have been employed. From here on, they will be referred to as $30 \mathrm{~N}$ and P18, respectively.

Each generator has been tested at a $4 \mathrm{kV}$ level as a function of:

- $\quad$ the DSO bandwidth;

- the cross-section and position of the ground strap cable

- the tripod type.

The measurements have been performed by means of a digital oscilloscope, LeCroy Wavepro 760Zi, which allows to set different analog bandwidths (up to $6 \mathrm{GHz}$ ), with a maximum single-shot sample rate of $40 \mathrm{GS} / \mathrm{s}$. The input impedance of the DSO has been set at $50 \Omega$, as required by the standards. The measurement chain has been placed inside a shielded chamber.

The reference situation, shown in the following figures, is the one specified in the Annex B of the IEC standard.

For the sake of brevity, only the most significant results will be shown in the next sections.

\section{A. Effect of the DSO bandwidth}

The figures show the influence of different DSO bandwidth values, mostly on the current peak value and rise time. The two generators have been tested in the reference situation, with a DSO bandwidth ranging from $1 \mathrm{GHz}$ to 6 GHz. The percentage deviations between the minimum and the maximum current peaks have been found equal to $3 \%$ for the $30 \mathrm{~N}$ gun and $10 \%$ for the P18 gun, as shown in Fig. 5 and Fig.6, where the peak differences have been enlarged.

It can be discerned that a wider bandwidth of the DSO would allow critical situations to be detected (values close to the immunity limits), which may influence the calibration result. 


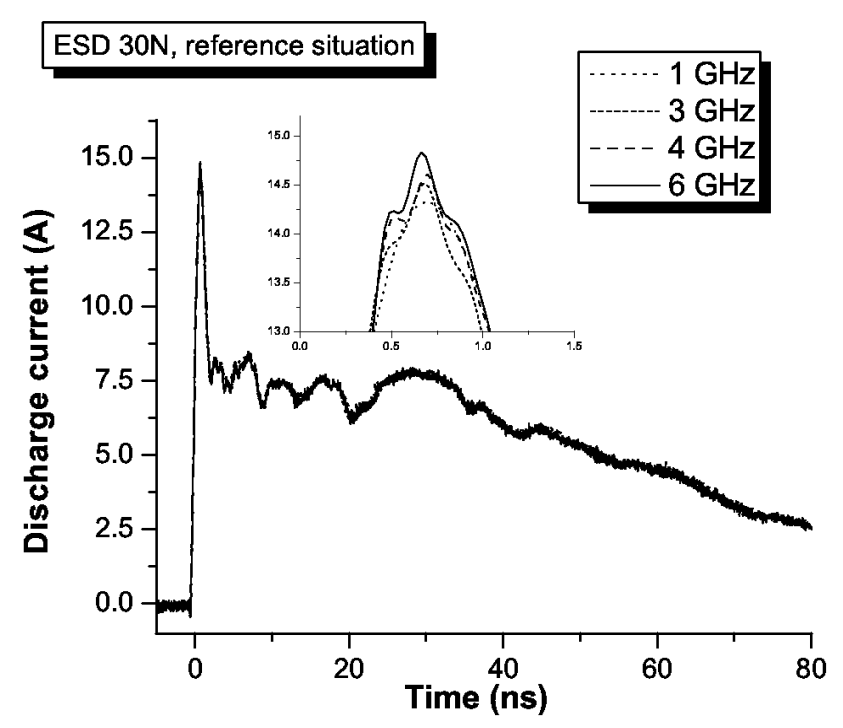

Fig.5. Influence of the bandwidth level on the peak value of the discharge current. The ESD generator used is $30 \mathrm{~N}$.

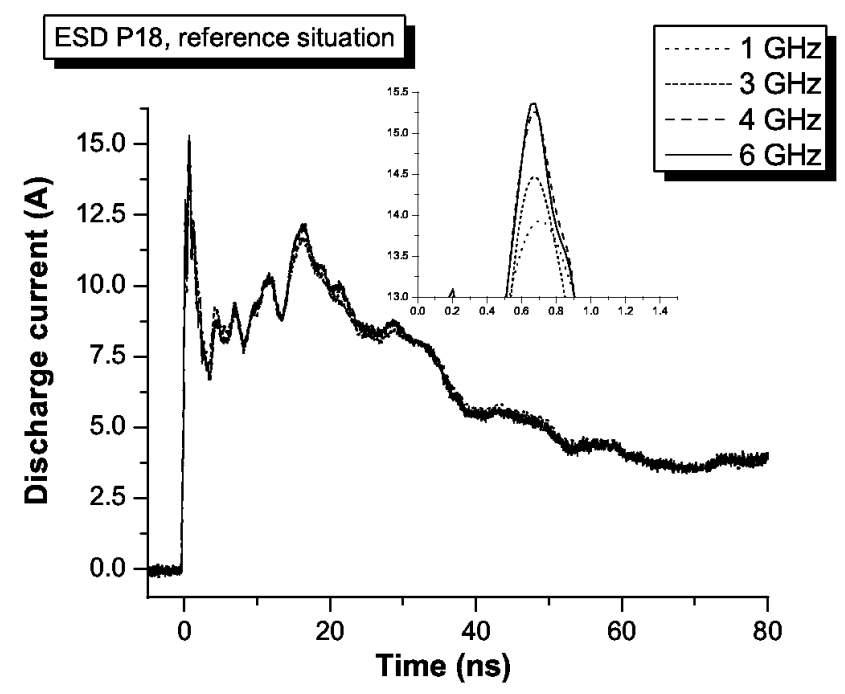

Fig.6. Influence of the bandwidth level on the peak value of the discharge current. The ESD generator used is P18.

\section{B. Effect of the ground strap cross-section}

In order to investigate the influence of the ground strap cross-section, measurements have been performed by using a circular cross-section cable of small radius (called thin) and a rectangular cross-section flat cable (called flat) as the ground strap (Fig.7). Both cables have a $2 \mathrm{~m}$ length and are connected to the same position on the vertical plane. The bandwidth of the DSO has been set at $6 \mathrm{GHz}$.

It should be noted how the choice of the ground strap strongly modifies the current waveform. Furthermore, the standards do not specify any requirement except for the cable length $((2 \pm 0.05) \mathrm{m})$.

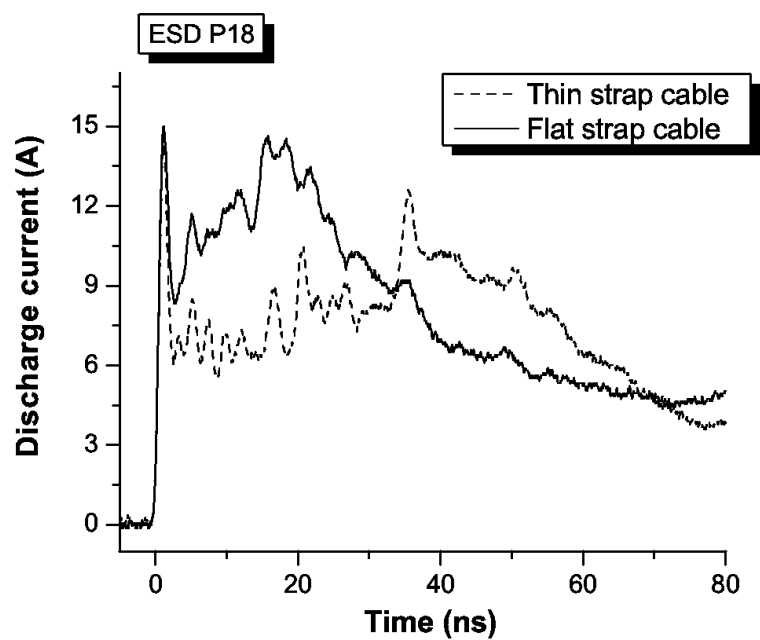

Fig.7. Influence of the ground strap cable cross-section on the current waveform. The ESD generator used is P18.

\section{PRELIMINARY MODELLING APPROACH}

With regard to the CD calibration set-up, a great effort has been made in the implementation of a suitable noncommercial numerical model, which allows computing and identifying optimal set-up configurations, in order to improve the measurement repeatability and reproducibility.

In the literature, some authors have proposed both circuital and full-wave ESD generator models [3],[9],[10],[11]. To assure model accuracy, a key issue is the accurate computation of those stray distributed parameters which strongly affect the discharge current waveform, in particular the current tail.

A preliminary analysis of some stray parameter effects on the ESD waveform can be carried out through the circuital model [3], sketched in Fig.8:

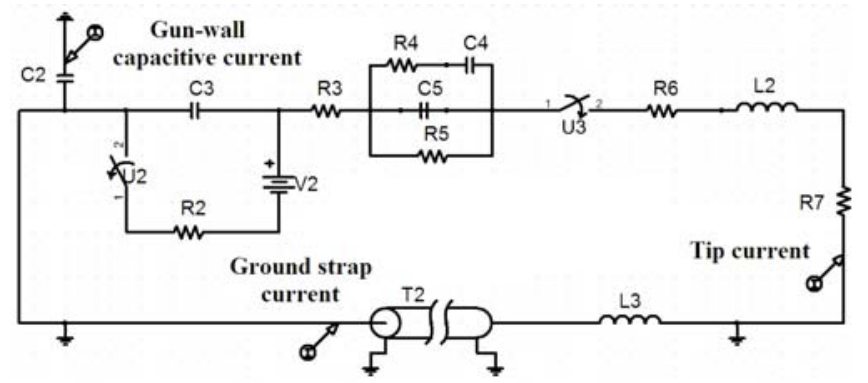

Fig.8. Circuital model of the ESD generator and the experimental set-up (CD method).

In order to investigate the sensitivity of the current waveform to the stray parameters, it is interesting to analyze different circuital situations, in which one or more parameters are changed within an appropriate range. The resulting current waveforms are then compared with the IEC reference current. The following figures highlight the strong sensitivity of the current waveform to the gun-wall capacitance (Fig.9), and to the characteristic parameters (time delay and characteristic impedance) of the ground strap cable modeled as a transmission line (Fig.10). 


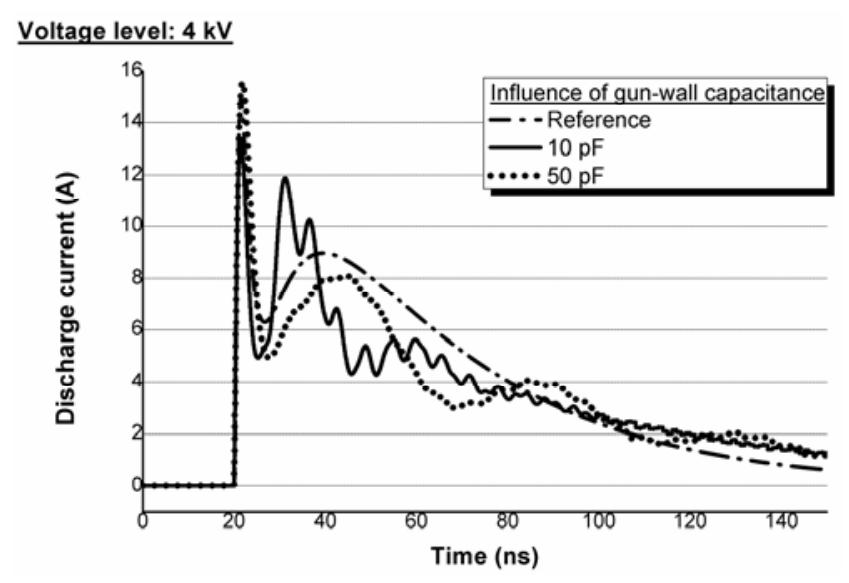

Fig.9. Sensitivity of the current waveform to the gun-wall capacitance.
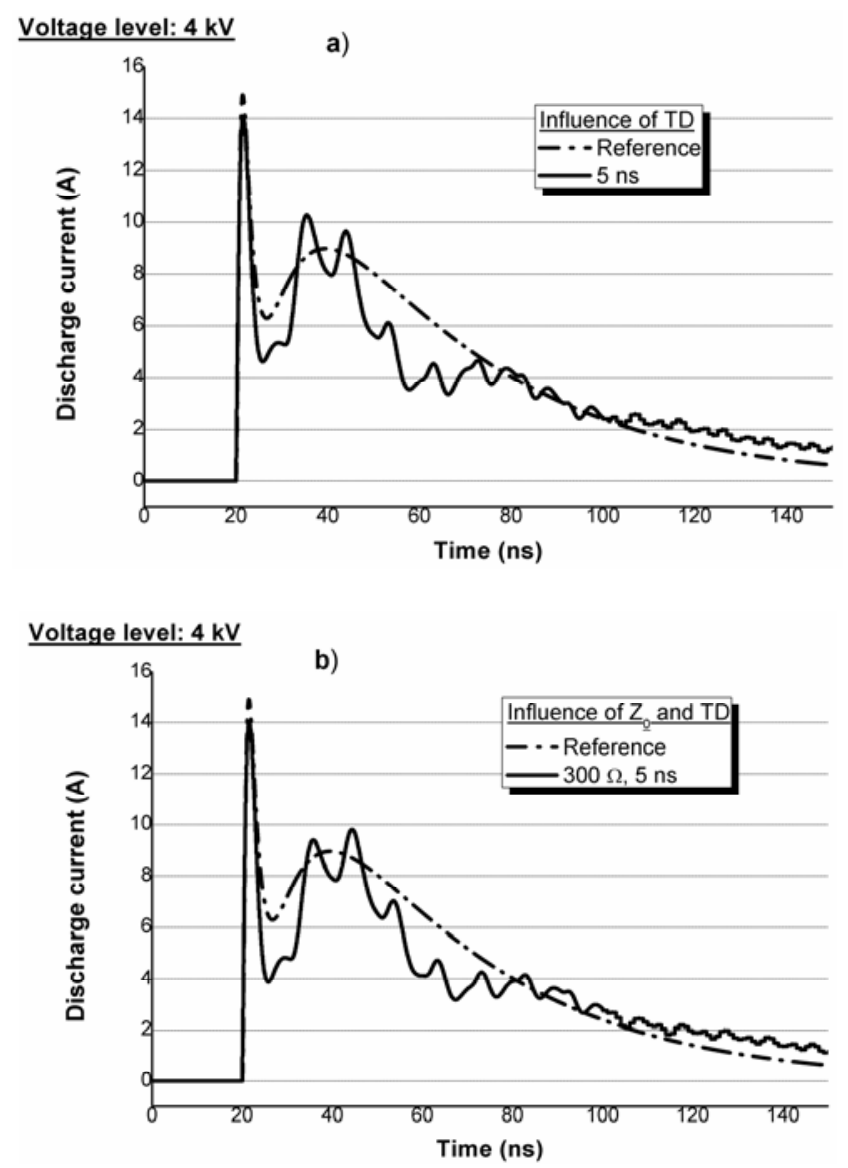

Fig.10. Sensitivity of the current waveform to the ground strap parameters: a) time delay b) characteristic impedance.

As in the results of the measurements, the current waveform in Fig.9 shows the presence of a second high peak, while the other figures underline the effects on the tail behavior, in particular referring to the $I_{30}$ and $I_{60}$ parameters. However, the approximated approach of the circuital model does not allow an exhaustive and complete modeling analysis of the whole distributed system (ESD generator + ground strap + metallic wall), in particular as concerns the investigation of the electromagnetic behavior in the high part of the frequency spectrum. Therefore, our aim is to develop a more accurate modeling approach in the near future.

\section{CONCLUSIONS}

The paper deals with many critical aspects of the ESD generator calibration process in order to convey ideas and advice for the procedure improvement. The topic is developed by separately discussing the contact discharge and the air discharge calibration methods.

On the basis of the obtained results, some useful conclusions and practical suggestions can be used by both the scientific community and the laboratory technicians.

As concerns the contact discharge method, the following issues might be considered during the review process of the IEC and ISO standards:

1. The bandwidth of DSO should be enlarged to values greater than $2 \mathrm{GHz}$, to detect the first current peak with higher accuracy.

2. The current waveform and especially the current value at $60 \mathrm{~ns}$ can significantly vary as a function of the used ground strap. As well as the cable length, also its cross-section and size play an important role in stating the features of the waveform. It should be desirable to make the measurement outcome as independent as possible of the cable geometry.

3. The possibility to carry out a calibration procedure with the support of suitable and accurate numerical methods, giving rise to a hybrid experimentalnumerical approach to verify the actual performance of the ESD generator should be considered.

As far as the air discharge mode is concerned, on the basis of the outcomes presented in Section 3, the proposed calibration scheme can be considered suitable to fulfill the voltage requirements of the ESD standards.

\section{REFERENCES}

[1] EC Std. (2004). EMC Directive 204/108/EC.

[2] Borsero, M., Caniggia, S., Sona, A., Stellini, M., Zuccato, A. (2008). A new proposal for the uncertianty evaluation and reduction in air electrostatic discharge tests. In EMC Europe 2008: International Symposium on Electromagnetic Compatibility, 8-12 September 2008, Hamburg, Germany, 1-6.

[3] Caniggia, S., Maradei, F. (2006). Circuit and numerical modelling of electrostatic discharge generators. IEEE Trans. Ind. Appl., 42, 1350-1357.

[4] IEC Std. (2008). Electromagnetic Compatibility (EMC) - Part 4-2: Testing and Measurement Techniques-Electrostatic Discharge Immunity Test. IEC 61000-4-2.

[5] ISO Std. (2008). Road Vehicles-Test Methods for Electrical Disturbances from Electrostatic Discharges. ISO 10605.

[6] Hilty, K., Ryser, H., Herrmann, U. (2001). Calibration of electrostatic discharge generators and results of an international comparison. IEEE Trans. Instrum. Meas., 50, 414-418, 2001. 
[7] Baran, J., Sroka, J. (2010). Distortion of ESD generator pulse due to limited bandwidth of verification path. IEEE Trans. Electromagn. Compat., 52 (4), 797-803.

[8] Borsero, M., Sardi, A., Vizio, G. (2008). Calibration methods for electrostatic discharge generators. In CPEM 2008: Conference on Precision Electromagnetic Measurements Digest, 8-13 June 2008. IEEE, 504-505.

[9] Wang, K., Pommerenke, D., Chundru, R., Van Doren, T, Drewniak, J.L., Shashindranath, A. (2003). Numerical modelling of electrostatic discharge generators. IEEE Trans. Electromagn. Compat., 45 (2), 258-271.
[10] Zaridze, R., Karkashadze, D., Djobava, R.G. Pommerenke, D., Aidam, M. (1996). Numerical calculation and measurement of transient fields from electrostatic discharges. IEEE Trans. Compon. Pack. Man. C, 19, 178-183.

[11] Caniggia, S., Maradei, F. (2007). Numerical prediction and measurement of ESD radiated fields by free-space field sensors. IEEE Trans. Electromagn. Compat., 49, 494-503.

Received December 6, 2010. Accepted March 22, 2011. 